PROCEEDINGS OF THE

AMERICAN MATHEMATICAL SOCIETY

Volume 125, Number 6, June 1997, Pages 1831-1833

S 0002-9939(97)03710-6

\title{
J-HOLOMORPHIC CURVES IN ALMOST COMPLEX SURFACES DO NOT ALWAYS MINIMIZE THE GENUS
}

\author{
G. MIKHALKIN
}

(Communicated by Ronald Stern)

\begin{abstract}
The adjunction formula computes the genus of an almost complex curve $F$ embedded in an almost complex surface $M$ in terms of the homology class of $F$. If $M$ is Kähler (or at least symplectic) and the self-intersection of $F$ is non-negative then the genus of any other surface embedded in $M$ and homologous to $F$ is not less then the genus of $F$ (the proof of this statement (which is a generalization of the Thom conjecture for $\mathbb{C} P^{2}$ ) was recently given by the Seiberg-Witten theory). This paper shows that the extra assumptions on $M$ are essential for the genus-minimizing properties of embedded almost complex curves.
\end{abstract}

Let $M$ be a connected smooth 4-manifold with the tangent bundle $\tau M$ equipped with a fiberwise-linear map $J: \tau M \rightarrow \tau M$ respecting the fibers and such that $J^{2}=-1$. In this case $M$ is called an almost complex surface. The $\mathbb{R}$-linear map $J$ makes $\tau M$ into a 2-dimensional complex bundle and induces an orientation on $M$. The canonical class $K \in H^{2}(M ; \mathbb{Z})$ is the Euler class of the exterior square over $\mathbb{C}$ of $\tau M$ multiplied by $(-1)$.

An embedded surface $F \subset M$ is called a $J$-holomorphic curve if its tangent bundle $\tau F$ is invariant under $J$. A $J$-holomorphic curve gets an orientation from $J$. If $F$ is $J$-holomorphic then the normal bundle $\nu F$ can be chosen to be invariant under $J$, and the direct sum formula for the characteristic classes of bundles produces the adjunction formula for the genus $g(F)$ of $F$ :

$$
g(F)=1+\frac{F \cdot F+K \cdot F}{2}
$$

where $F . F$ denotes the self-intersection of $F$ and $K . F$ denotes the result of evaluation of $K$ on $F$.

Let $E \subset M$ be an orientable surface homologous to $F$. The genus $g(E)$ of $E$ is not determined by its homology class. However, in the case when $M$ is symplectic with the symplectic form $\omega$ compatible to $J$ so that $\omega(x, J x) \geq 0$ for any $x \in \tau M$ and $F . F$ is non-negative, the adjunction formula turns into the adjunction inequality

$$
g(E) \geq g(F)=1+\frac{F \cdot F+K . F}{2}
$$

recently proven by means of the Seiberg-Witten theory.

The following theorem shows that the adjunction inequality $g(E) \geq g(F)$ does not hold for all almost complex surfaces.

Received by the editors September 22, 1995.

1991 Mathematics Subject Classification. Primary 57R95, 53C15.

(C)1997 American Mathematical Society 
Theorem 1. There exist an almost complex surface $M$ and two smooth closed surfaces $E, F \subset M,[E]=[F] \neq 0 \in H_{2}(M)$, such that $F$ is $J$-holomorphic and $g(E)<g(F)$.

Proof. Let $M$ be diffeomorphic to $\mathbb{C} P^{2} \# \mathbb{C} P^{2} \# \mathbb{C} P^{2}$. This manifold admits an almost complex structure with the canonical class $-(3,3,1) \in \mathbb{Z} \oplus \mathbb{Z} \oplus \mathbb{Z}=H^{2}(M)$ by Satz 4.6 of [1], since $(3,3,1) \cdot(3,3,1)=19=2 \chi(M)+3 \sigma(M)$. The next two lemmata produce the required $E$ and $F$.

Lemma 1. There exists a deformation of the almost complex structure on $M$ such that $(4,0,0) \in H_{2}(M)$ is realizable by a closed $J$-holomorphic curve $F$ of genus 3 .

Proof. Let $F \subset M$ be the orientable surface of genus 3 produced by an embedding of a nonsingular quartic curve into the first summand $\mathbb{C} P^{2}$ of $M \approx \mathbb{C} P^{2} \# \mathbb{C} P^{2} \# \mathbb{C} P^{2}$. We may assume (after a small deformation of $F$ ) that $F$ is $J$-holomorphic in a neighbourhood of a point $x \in F$.

The restriction $\left.T M\right|_{F}$ of the tangent bundle of $M$ is a trivial 4-dimensional bundle (since $w_{1}\left(\left.T M\right|_{F}\right)=0$ and $w_{2}\left(\left.T M\right|_{F}\right)=0$ ). Choose a trivialization of $\left.T M\right|_{F}$. The subbundle $\left.T F \subset T M\right|_{F}$ is then given by a map $\alpha: F \rightarrow G_{4,2}$, where $G_{4,2} \approx S^{2} \times S^{2}$ is the Grassmannian of the orientable 2-subspaces in $\mathbb{R}^{4}$. The homotopy class of such a map is determined by two numbers corresponding to the Euler number of the bundle and the Euler number of the normal bundle.

The $J$-invariant planes passing through a section of $\left.T F\right|_{F-\{x\}}$ determine a complex subbundle $\left.B \subset T M\right|_{F}$ with the first Chern number $c_{1}(B)=\chi(F)=-4$. The first Chern number of the bundle normal to $B$ is $16=F . F$, since $c_{1}\left(\left.T M\right|_{F}\right)=$ $(3,3,1) \cdot(4,0,0)=12$. Therefore, the map $\beta: F \rightarrow G_{4,2}$ corresponding to $B$ is homotopic to $\alpha$.

To finish the proof we deform the almost complex structure in the tubular neighbourhood of $F$ by following the homotopy between $\alpha$ and $\beta$ to make TF $J$-invariant.

Lemma 2. There exists an orientable surface $E \subset M$ of genus 1 realizing the homology class $(4,0,0) \in H_{2}\left(\mathbb{C} P^{2} \# \mathbb{C} P^{2} \# \mathbb{C} P^{2}\right)$.

Remark. By the Rokhlin-Hsiang-Szczarba inequality [5], [2] the genus of any embedded surface of homology class $(4,0,0)$ is at least 1 .

Proof of Lemma 2. A nonsingular quartic curve $C \subset \mathbb{C} P^{2}$ can be obtained from a union of 4 lines and, therefore, it admits a $(-1)$-membrane for each of its 3 handles (i.e. a disk $M \subset \mathbb{C} P^{2}$ normal to $C$ along the curve $M \cap C=\partial M$ coinciding with the cocore of the handle and such that the self-intersection number of $M$ equipped with the framing on $\partial M$ coming from $C$ is -1 ). Making a connected sum with the pair $\left(\mathbb{C} P^{2}, \mathbb{C} P^{1}\right)$ (cf. [4]) allows us to make the self-intersection of the membrane into zero and, thus, make an embedding surgery removing the handle along this membrane. After repeating this procedure two times we get the required surface $E \subset \mathbb{C} P^{2} \# \mathbb{C} P^{2} \# \mathbb{C} P^{2}=M$ of genus 1 (cf. getting a $(3,0)$-sphere in $\mathbb{C} P^{2} \# \mathbb{C} P^{2}$ in $[3])$.

Acknowledgment. I am indebted to Y. Eliashberg for the discussion which led to the proof of Lemma 1.

Remark. As I was informed by the referee, an example similar to the one presented in this paper was given by D. Kotschick in a lecture in Cambridge in 1994. 


\section{REFERENCES}

[1] F. Hirzebruch, H. Hopf, Felder von Flächenelementen in 4-dimensionalen Mannigfaltigkeiten, Math. Ann. 136 (1958), 156-172. MR 20:7272

[2] W. Hsiang, R. Szczarba, On embedded surfaces in four-manifolds, Proc. Sympos. Pure Math. 22 (1970), 97-103. MR 49:4000

[3] M. Kervaire, J. Milnor, On 2-spheres in 4-manifolds, Proc. Nat. Acad. Sci. U.S.A. 47 (1961), 1651-1657. MR 24A:2968

[4] G. Mikhalkin, Surfaces of small genus in connected sums of $\mathbb{C} P^{2}$ and real algebraic curves with many nests in $\mathbb{R} P^{2}$, Contemp. Math. 182 (1995), 73-82. MR 95m:57050

[5] V. A. Rokhlin, Two-dimensional submanifolds of four-dimensional manifolds, Funktsional. Anal. i Prilozhen. 5(1) (1971), 48-60. MR 45:7733

Department of Mathematics, University of Toronto, Toronto, Ontario, Canada M5S $1 \mathrm{~A} 1$

E-mail address: mihalkin@math.toronto.edu 